\title{
Wide-Field-of-View Atom Probe Reconstruction
}

\author{
B. P. Geiser, D. J. Larson, E. Oltman, S. Gerstl, D. Reinhard, T. F. Kelly, and T. J. Prosa \\ Imago Scientific Instruments Corp., 5500 Nobel Dr., Madison, WI 53711
}

In atom probe tomography, it is usually desirable to obtain the largest possible field of view (FOV) in the analysis and recent advances in instrumentation [1] have made significant increases in FOV. However, the most commonly used data reconstruction techniques were designed for much smaller FOV instruments and as such, the small-angle approximations employed are less accurate for the current generation of instruments. Prior to the advent of wide FOV instruments, the geometric assumptions described by Blavette [2], and later applied by Bas [3], were widely considered the standard global reconstruction technique [4]. This model incorporates a simple point projection to account for lateral magnification and uses geometric models of the global tip shape to reconstruct depth information. It also assumes that the original shape of acquired volumes is small enough in lateral extent to be considered cylindrical and the radius of the tip is determined atom-by-atom by the specimen voltage. In the early reconstructions [3], the actual shank angle is ignored and it is assumed to be zero in the calculation of the volume increment. In using the voltage as a proxy for the tip radius it will often be the case that the reconstructed geometry is not conical, and indeed may be extremely irregular. This can happen for instance in a multi-layer system where evaporation fields are rapidly changing. In this case the assumption of a fixed evaporation field is clearly erroneous and induces rapidly changing model geometry.

In advancing the reconstruction procedure for wide FOV, a methodology that does not make small angle assumptions is used. A point projection method similar to that of Walck [5] is used as the starting point, followed by decoupling the evolution of the tip radius from the specimen voltage with new formulations developed for magnification and volume increment per reconstructed atom. The complete analytic volume of the assumed model is calculated (Fig. 1), keeping all terms and obtaining an exact expression for volume increment as a function of tip radius, shank angle and instrument geometry. Similarly, an exact expression for magnification in this model is obtained.

Quantitative comparisons are made (Fig. 2-3) for three distinct reconstruction algorithms: a) small FoV, voltage-evolution; b) large FoV, voltage-evolution; and c) large FoV, fixed-shank angle.

\section{References}

[1] T. Kelly, et al., Rev. Sci. Instrum. 78 (2007) 031101.

[2] D. Blavette, et al., Revue Phys. Appl. 17 (1982) 435.

[3] P. Bas, et al., Appl. Surf. Sci. 87/88 (1995) 298.

[4] M. Miller, "Atom Probe Tomography: Analysis at the Atomic Level" Kluwer Academic/Plenum Publishers, New York, 2000.

[5] S. D. Walck, et al., Journal de Phys. 47-C2 (3) (1986) 451. 


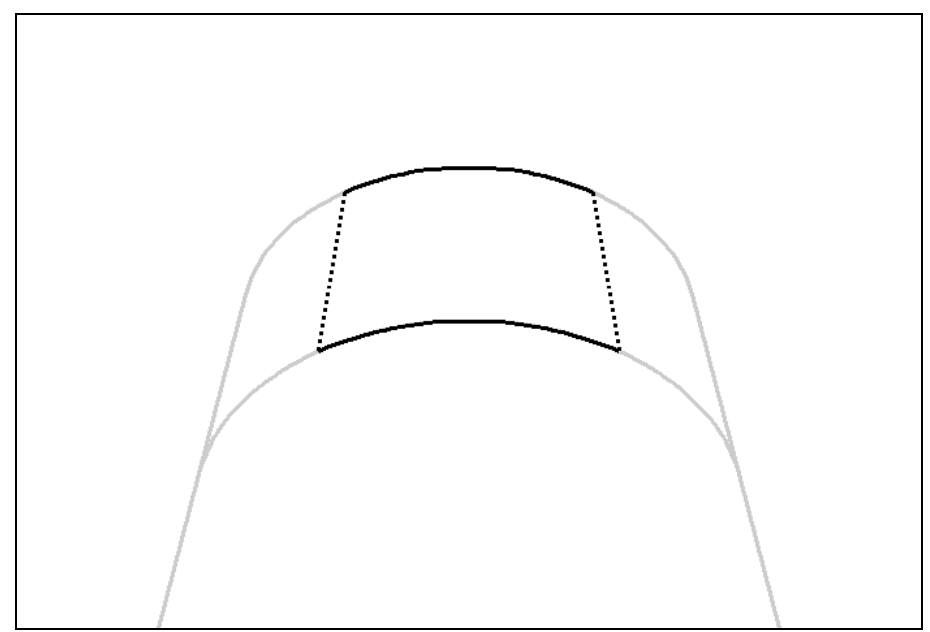

Fig. 1. Schematic illustration of evaporation (gray) and analysis (black) geometries. Tangential continuity of the apex with the shank is assumed for the model.

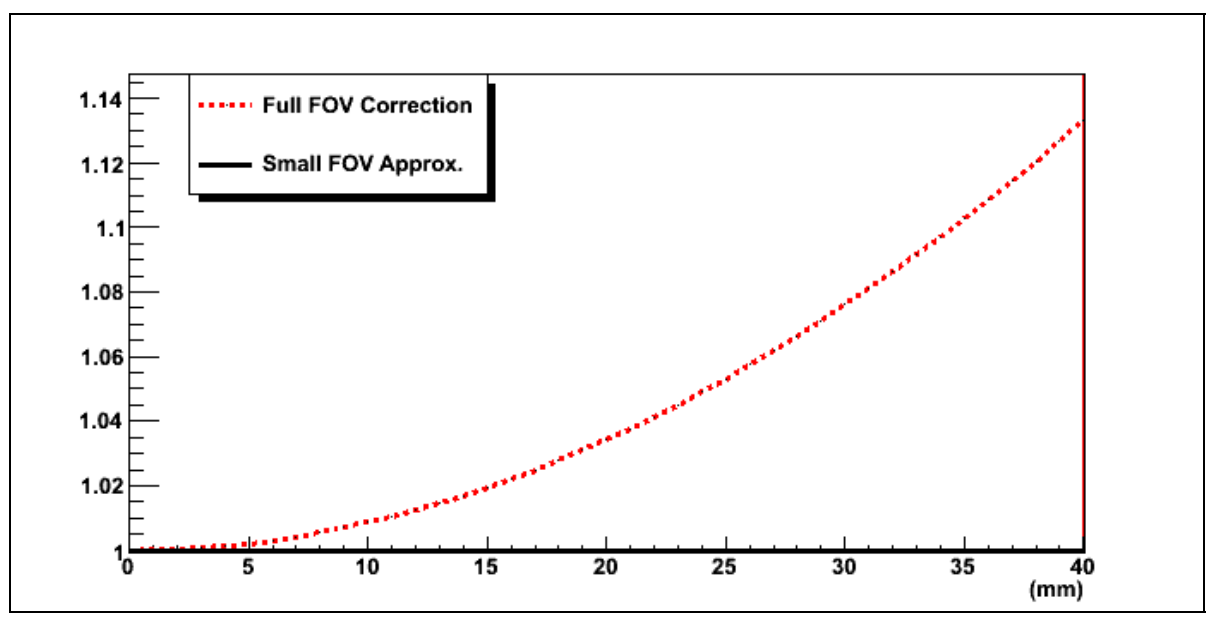

Fig. 2. Relative magnification change between small FoV and large FoV fixed-shank reconstructions as a function of hit-position on the detector.

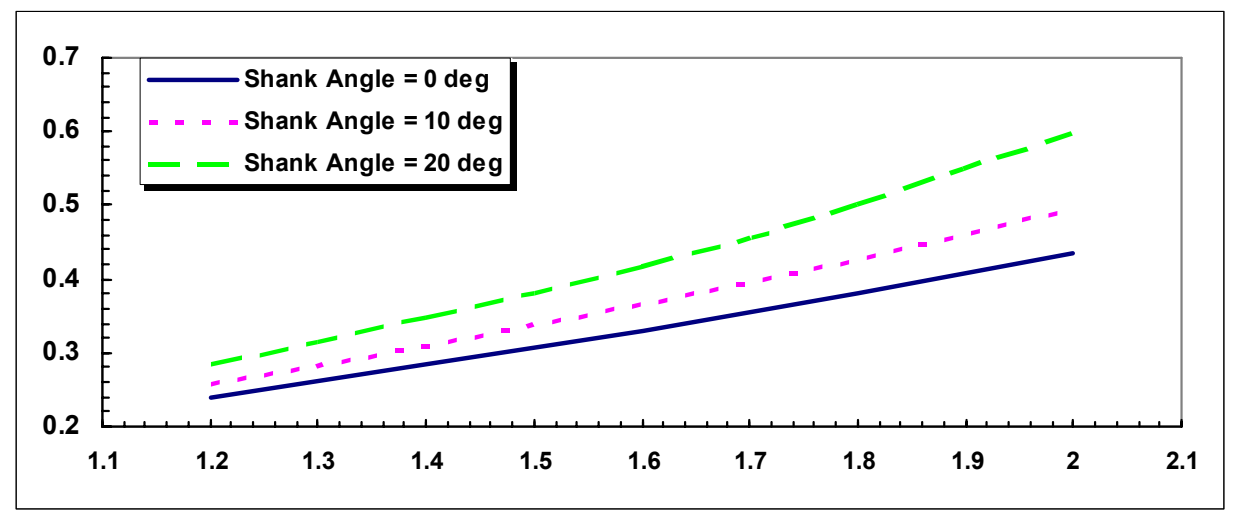

Fig. 3. Relative difference in the volume increment per reconstructed atom (\%) between small FoV and large FoV fixed-shank reconstructions. Differences are plotted as a function of image compression factor for three different specimen shapes. 